\title{
Production of exotic charmonium in heavy ion collisions
}

\author{
F. S. Navarra ${ }^{1, a}$ \\ ${ }^{1}$ Instituto de Física, Universidade de São Paulo, Rua do Matão 1371, 05508-090, São Paulo, SP, Brazil
}

\begin{abstract}
We present a short review of exotic charmonium production in heavy ion collisions. We discuss predictions for the production cross section of several of these states in ultra-peripheral $\mathrm{Pb}-\mathrm{Pb}$ collisions at the LHC. The experimental study of these processes is feasible and can be used to yield valuable information about the structure of multiquark states. We also address $X(3872)$ production in central $\mathrm{Pb}-\mathrm{Pb}$ collisions. In particular, we discuss the suppression of $X(3872)$ during the hadron gas phase. Finally, we comment on the very recent CMS data on the $X(3872)$ yield in $\mathrm{Pb}-\mathrm{Pb}$ collisions.
\end{abstract}

\section{Introduction}

During the past decade, the existence of exotic hadrons has been firmly established $[1,2]$. These hadrons are not simply made by quark-antiquark or by three quarks. They are genuine multiquark states but we do not know yet how they are organized. Among the proposed configurations, the meson molecule and the tetraquark are the most often discussed. A tetraquark is compact and the interaction between the constituents occurs through color exchange forces whereas a meson molecule is an extended object and its constituents are weakly bound by meson exchange. In principle the new exotic states can be tetraquarks, meson molecules or tetraquark-molecule mixtures. Indeed this mixed approach has led to the best description of the $X(3872)$. In Ref. [3] the mass and strong decay width were very well reproduced assuming that the $X(3872)$ has a $c \bar{c}$ component with a weight of $97 \%$ and a $D \bar{D}^{*}$ component with $3 \%$ weight. Even if the best description is given by a mixture it is still very important to understand the individual role played by each component.

The production of exotic particles in hadronic colliders is not yet well understood. It has been argued $[4,5]$ that it is difficult to produce molecules in p-p collisions. In a pure molecular approach the cross section for $X(3872)$ production estimated in $[4,5]$ is two orders of magnitude smaller than the measured one. However, in [6] the procedure to obtain these estimates was criticized and the molecular picture was reconciled with data. In Ref. [7] it was shown that the best description can be achieved with a charmonium-molecule combination, i.e. $\chi_{c 1}^{\prime}-D \bar{D}^{*}$, in which the $c \bar{c}$ component is of the order of $28-44 \%$. An attempt to explain the data in the tetraquark approach was performed in [8].

Relativistic heavy ion collisions offer a new testing ground for the ideas about the structure of exotic charmonium states. In ultra-peripheral collisions the photon flux is extremely high and one can look for states $(\mathrm{X})$ produced through photon-photon fusion $(\gamma \gamma \rightarrow X)$ in a very clean environment. In central collisions the number of produced charm quarks is very large and so is the expected exotic

\footnotetext{
a e-mail: navarra@if.usp.br
} 
charmonium yield. In the next section we briefly review the existing results on the exotic charmonium production cross section in ultra-peripheral collisions. In the following section we focus on the production of $X(3872)$ in central collisions. We then give a summary of the situation and try to list the next steps in the field.

\section{Ultra-peripheral heavy ion collisions}

The strong electromagnetic fields generated by these ions allow the production of a meson in photonphoton interactions. The idea of studying exotic meson production in UPCs was pioneered in [9]. Later, in Ref. [10], the same formalism was applied to the production of mesons and exotic states in proton-proton collisions. In [11] these calculations were updated and extended to proton-proton and nucleus-nucleus collisions at LHC energies, focusing on photon-photon production of the heavy exotic charmonium states and including $X(3915), Z(3930)$ and $X(4160)$. All the ingredients of these calculations are fixed with the exception of the two-photon decay width of the exotic state. In principle, tetraquark and molecular configurations would yield quite different numbers for the decay widths, which would yield quite different production cross sections. The two-photon decay width of the exotic states has been calculated in the molecular approach in several works [12]. Unfortunately the theoretical predictions of the tetraquark model are not yet available.

In [11] all the charmonium states were considered for which either a measurement or a theoretical estimate of the decay width is available. Moreover, the two possible assignments, $0^{++}$and $2^{++}$, for the states $X(3940)$ and $X(4140)$ were considered.

\begin{tabular}{|c|c|c|c|c|c|c|}
\hline State & \multirow{2}{*}{ Mass } & $\Gamma_{\gamma \gamma}^{\text {theor }}(\mathrm{keV})$ & \multicolumn{2}{|c|}{$\sigma_{b_{\min }}(\mu \mathrm{b})$} & \multicolumn{2}{|c|}{$\sigma_{F}(\mu \mathrm{b})$} \\
\cline { 4 - 7 } & & & $2.76 \mathrm{TeV}$ & $5.5 \mathrm{TeV}$ & $2.76 \mathrm{TeV}$ & $5.5 \mathrm{TeV}$ \\
\hline \hline $\mathrm{X}(3940), 0^{++}$ & 3943 & 0.33 & 4.2 & 8.2 & 6.5 & 11.8 \\
$\mathrm{X}(3940), 2^{++}$ & 3943 & 0.27 & 17.2 & 33.6 & 26.5 & 48.4 \\
$\mathrm{X}(4140), 0^{++}$ & 4143 & 0.63 & 6.5 & 12.9 & 10.2 & 18.7 \\
$\mathrm{X}(4140), 2^{++}$ & 4143 & 0.50 & 26.0 & 51.2 & 40.3 & 74.3 \\
$\mathrm{Z}(3930), 2^{++}$ & 3922 & 0.083 & 5.4 & 10.5 & 8.3 & 15.2 \\
$\mathrm{X}(4160), 2^{++}$ & 4169 & 0.363 & 18.4 & 36.4 & 28.6 & 52.7 \\
$\mathrm{Y}_{p}(3912), 2^{++}$ & 3919 & 0.774 & 50.5 & 98.6 & 77.9 & 142.2 \\
$\mathrm{X}(3915), 0^{++}$ & 3919 & 0.20 & 2.6 & 5.1 & 4.0 & 7.34 \\
\hline
\end{tabular}

Table 1. Cross sections for exotic meson production in $\mathrm{PbPb}$ collisions using the theoretical decay rates published in Ref. [12]. $\sigma_{b_{\min }}$ and $\sigma_{F}$ refer to different assumptions for the nuclear form factors.

In Table 1 we show the predictions for the cross sections for the production of several exotic mesons in $\mathrm{Pb}-\mathrm{Pb}$ collisions at $\sqrt{s}=2.76 \mathrm{TeV}$ and $\sqrt{s}=5.5 \mathrm{TeV}$ using two types of nuclear form factor. The cross section is enhanced by a factor $Z^{4}$ in $\mathrm{Pb}-\mathrm{Pb}$ collisions. This is reflected in the results, with the cross sections ranging from a few hundred nb up to a few tens of $\mu \mathrm{b}$.

In order to illustrate the behavior of the cross section with the collision energy $\sqrt{s}$ we take the state $X(3915)$ as example. Fig. 1a shows the behavior of the production cross section in $\mathrm{Pb}-\mathrm{Pb}$ collisions with $\sqrt{s}$ from $10 \mathrm{GeV}$ to $100 \mathrm{TeV}$. As it can be seen, this cross section can reach large values at energies covered by the future colliders such as the FCC (Future Circular Collider) and the CEPC - SPPC (Circular Electron Positron Collider - Super Proton Proton Collider). Fig. 1b, shows the rapidity distribution of the $X(3915)$ in $\mathrm{Pb}-\mathrm{Pb}$ collisions at $\sqrt{s}=5.5 \mathrm{TeV}$. The width of the rapidity distributions and the total cross sections are the most useful quantities to be compared with theoretical predictions of particle production in UPCs in relativistic heavy ion collisions. 


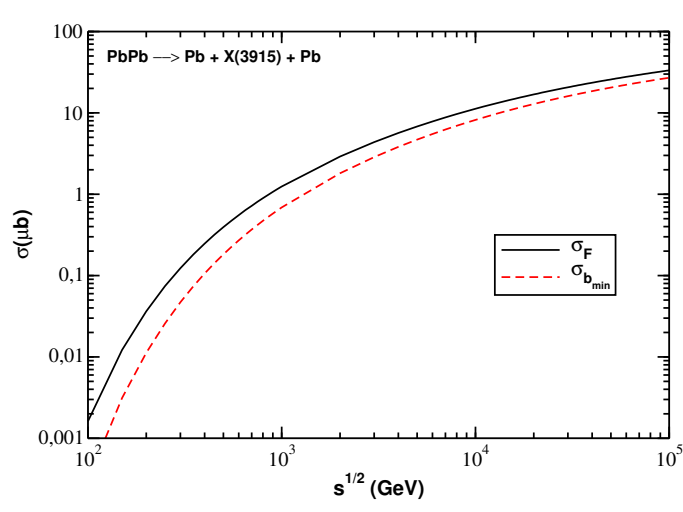

(a)

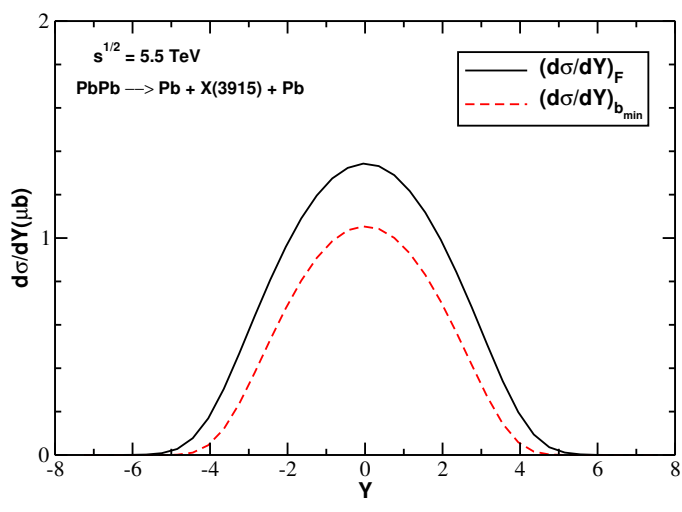

(b)

Figure 1. a) Cross section of the process $P b P b \rightarrow X(3915)$ as a function of the energy $\sqrt{s}$. b) Rapidity distribution of the produced $X(3915)$ with the same definitions as in a). Figure taken from Ref. [11].

\section{Central heavy ion collisions}

As pointed out by the EXHIC Collaboration [13], high energy heavy ion collisions offer an interesting scenario to study the production of multiquark states. In these processes, a quite large number of heavy quarks is expected to be produced. Moreover, the formation of quark gluon plasma (QGP) may enhance the production of exotic charmonium states, since the charm quarks are free to move over a large volume and they may coalesce to form bound states at the end of the QGP phase.

One of the main conclusions of Refs. [13] was that, if the production mechanism is coalescence, then the production yield of these exotic hadrons at the moment of their formation strongly reflects their internal structure. In particular it was shown that in the coalescence model the production yield of the X(3872), at RHIC or LHC energies, is almost 20 times bigger for a molecular structure than for a tetraquark configuration. After being produced at the end of the quark gluon plasma phase, the $X(3872)$ interacts with other hadrons during the expansion of the hadronic matter. Therefore, the $X(3872)$ can be destroyed in collisions with the comoving light mesons, such as $X+\pi \rightarrow \bar{D}+D$, $X+\pi \rightarrow \bar{D}^{*}+D^{*}$, but it can also be produced through the inverse reactions, such as $D+\bar{D} \rightarrow X+\pi$, $\bar{D}^{*}+D^{*} \rightarrow \pi+X$. We expect these cross sections to depend on the spatial configuration of the $X(3872)$.

The interactions of the $X$ in a hadronic medium were studied in the framework of $S U(4)$ effective Lagrangians [14]. The authors computed the corresponding production and absorption cross sections, finding that the absorption cross section is two orders of magnitude larger than the production one. In particular, for the largest thermally averaged cross sections they find: $<\sigma v>_{\pi X \rightarrow D^{*} \bar{D}^{*}} \simeq 30<$ $\sigma v>_{D^{*} \bar{D}^{*} \rightarrow \pi X}$. In spite of this difference, the authors of [14] arrived at the intriguing conclusion that the number of $X$ 's stays approximately constant during the hadronic phase. Moreover, the terms with anomalous couplings were not included in the calculations. In Ref. [15] the authors showed that

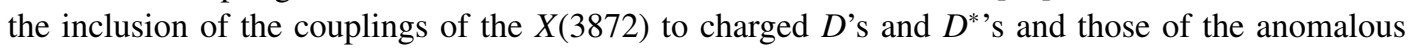
vertices, $\pi D^{*} \bar{D}^{*}$ and $X D^{*} \bar{D}^{*}$, increases the cross sections by more than one order of magnitude. These anomalous vertices also give rise to new reaction channels, namely, $\bar{D}+D^{*} \rightarrow \pi+X$ and $\pi+X \rightarrow$ $\bar{D}+D^{*}$. The changes that the above mentioned contributions can produce in the $X$ abundance (and in its time evolution) were evaluated in Ref. [16] and they are shown in Fig. 2. In the figure the solid line represents the result obtained in [14]. The dashed line shows the result when one includes the 
couplings of the $X(3872)$ to charged $D$ 's and $D^{*}$ 's. The light-shaded band shows the results obtained with the further inclusion of the diagrams containing the anomalous vertices. The band reflects the uncertainty in the $X \bar{D}^{*} D^{*}$ coupling constant, which is $g_{X \bar{D}^{*} D^{*}}=12.5 \pm 1.0$.

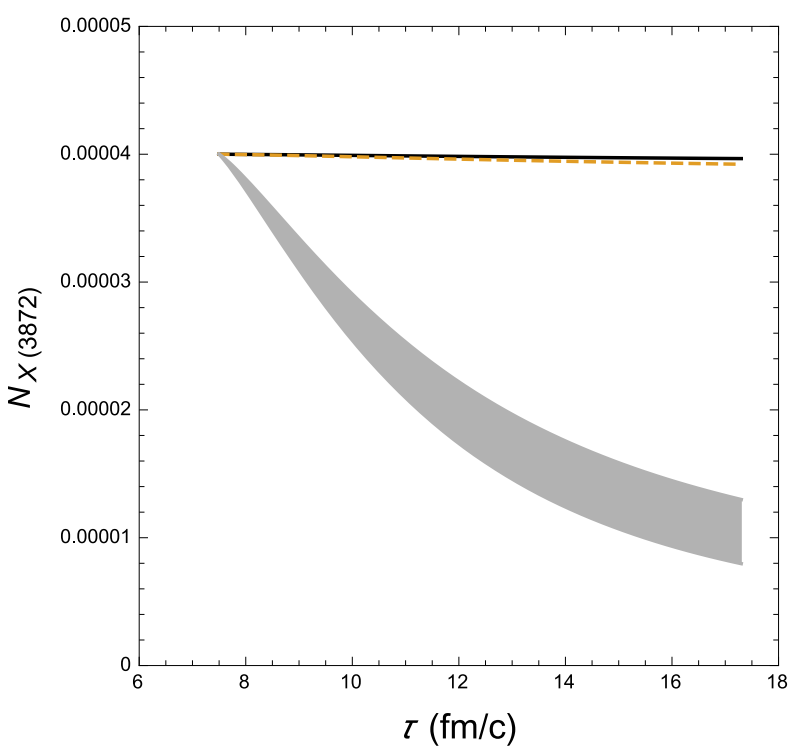

Figure 2. Time evolution of the $X(3872)$ abundance as a function of the proper time $\tau$ in central Au-Au collisions at $\sqrt{s_{N N}}=200 \mathrm{GeV}$. The solid line, the dashed line and the light-shaded region represent the results obtained

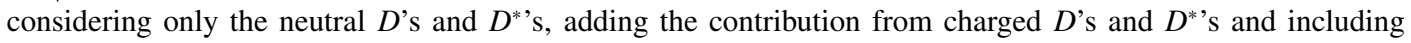
contributions from the anomalous vertices respectively. Figure taken from Ref. [16].

As can be seen, without the inclusion of the anomalous coupling terms, the abundance of $X$ remains basically constant. Including the coupling of the $X$ to charged $D$ 's and $D^{*}$ 's does not bring any important change in the time evolution of the $X$ abundance. On the other hand, the inclusion of the anomalous coupling terms modifies the behavior of the $X(3872)$ yield, producing a fast decrease of the $X$ abundance with time.

If the $X(3872)$ would be a tetraquark state it would be formed at the end of the QGP phase and would be massively destroyed during the hadron gas phase. If the $X(3872)$ would be a molecular state, it would be formed with an abundance 20 times larger at the end of the QGP phase. Moreover it would be also formed by meson coalescence at the end of the hadronic phase. According to [14], at this time the average number of $X$ 's, considering it as a $D \bar{D}^{*}$ molecule, is $N_{X(\text { mol })} \approx 7.8 \times 10^{-4}$, which is about 80 times larger than yield of a tetraquark state at the end of the hadronic phase (see Fig. 2). We can then conclude that the QGP contribution for the $X(3872)$ production (as a tetraquark state and from quark coalescence) after being suppressed during the hadronic phase, becomes insignificant at the end of the hadronic phase. On the other hand, if the $X(3872)$ is a molecular state it will be produced by hadron coalescence at the end of the hadronic phase.

In Ref. [16] it was argued that if the X(3872) were observed in a heavy ion collision it must have been produced at the end of the hadronic phase and, hence, it must be a molecular state. Apparently this seems to be the case! 
Very recently, the CMS Collaboration measured the ratio of $1.1 \pm 0.51$ (stat.) \pm 0.53 (syst.) for high - $p_{T}$ prompt $X(3872)$ and $\psi(2 S)$ produced in $\mathrm{Pb}+\mathrm{Pb}$ collisions at $\sqrt{s_{N N}}=5.02 \mathrm{TeV}$ [17]. The central value is about a factor of 10 larger than that in $\mathrm{p}+\mathrm{p}$ collisions. These data suggest that, not only the $X(3872)$ is observed in heavy ion collisions but its production rate is larger than expected. If this exciting new experimental information is confirmed, it will motivate a refinement of the existing calculations. If the existing theoretical results are confirmed, we will be led to the conclusion that the $X(3872)$ is a meson molecule or, at least, has a molecular component.

\section{Future Perspectives}

The recent measurement of the $X(3872)$ by CMS, will give great impulse to the search for exotic charmonium. Hopefully this will motivate the experimental searches for other exotic charmonium states both in central and ultra-peripheral collisions. From the theoretical side, it is clear that there are some urgent tasks. The first one is to conclude the study of $X(3872)$ production in proton-proton collisions. We need a reliable calculation of the $X(3872)$ production cross section in the tetraquark approach, we need a final assessment about the existing calculations with the molecular approach and also a confirmation of the results obtained with the mixture approach. We need a calculation of the two-photon decay width of the $0^{++}$and $2^{++}$charmonium exotic states in the tetraquark approach. With them we will be able to compare the preditions of both approaches for the production cross sections in UPCs. Finally, we need to subject the coalescence model to a rigorous scrutiny and confirm its predictions for the $X(3872)$ (and also for the other exotic states) abundancies in central heavy ion collisions. The near future looks very busy and exciting for the field.

\section{Acknowledgments}

This work has been supported by CNPq and FAPESP-Brazil.

\section{References}

[1] For a recent review: R. M. Albuquerque, J. M. Dias, K. P. Khemchandani, A. Martinez Torres, F. S. Navarra, M. Nielsen, C. M. Zanetti, J. Phys. G 46, 093002 (2019).

[2] M. Nielsen and F. S. Navarra, Mod. Phys. Lett. A 29, 1430005 (2014); M. Nielsen, F. S. Navarra and S. H. Lee, Phys. Rept. 497, 41 (2010); F. S. Navarra, M. Nielsen and S. H. Lee, Phys. Lett. B 649, 166 (2007).

[3] R. D. Matheus, F. S. Navarra, M. Nielsen and C. M. Zanetti, Phys. Rev. D 80, 056002 (2009).

[4] C. Bignamini, B. Grinstein, F. Piccinini, A. D. Polosa and C. Sabelli, Phys. Rev. Lett. 103, 162001 (2009).

[5] A. Esposito, A. L. Guerrieri, L. Maiani, F. Piccinini, A. Pilloni, A. D. Polosa and V. Riquer, Phys. Rev. D 92, 034028 (2015).

[6] E. Braaten, L. P. He and K. Ingles, Phys. Rev. D 100, no. 9, 094024 (2019)

[7] C. Meng, H. Han and K. T. Chao, Phys. Rev. D 96, 074014 (2017).

[8] F. Carvalho, E. R. Cazaroto, V. P. Gonçalves and F. S. Navarra, Phys. Rev. D 93, 034004 (2016).

[9] C.A. Bertulani, Phys. Rev. C 79, 047901 (2009).

[10] V. P. Goncalves, D. T. Da Silva and W. K. Sauter, Phys. Rev. C 87, 028201 (2013).

[11] B. D. Moreira, C. A. Bertulani, V. P. Goncalves and F. S. Navarra, Phys. Rev. D 94, 094024 (2016). 
[12] T. Branz, T. Gutsche and V. E. Lyubovitskij, Phys. Rev. D 80, 054019 (2009); Phys. Rev. D 82, 054010 (2010); T. Branz, R. Molina and E. Oset, Phys. Rev. D 83, 114015 (2011).

[13] S. Cho, et al., Prog. Part. Nucl. Phys. 95, 279 (2017).

[14] S. Cho and S. H. Lee, Phys. Rev. C 88, 054901 (2013).

[15] A. Martinez Torres, K. P. Khemchandani, F. S. Navarra, M. Nielsen, L. M. Abreu, Phys. Rev. D 90, 114023 (2014), [Erratum: Phys. Rev.D 93, 059902 (2016)].

[16] L. M. Abreu, K. P. Khemchandani, A. Martinez Torres, F. S. Navarra, M. Nielsen, Phys. Lett. B 761, 303 (2016).

[17] Z. Tang, arXiv:2002.1079 